\title{
Stress-strain state of earth dam under harmonic effect
}

\author{
Bakhtiyar Khusanov* and Ozodakhon Khaydarova \\ IMSS, Academy of Sciences of Rep. of Uzb., 100187 Tashkent, Uzbekistan
}

\begin{abstract}
Stress-strain state of earth dams under the effect of harmonic (seismic) load from the base of the dam is numerically studied in the paper. Rock fill dam has good culvert properties and its water saturation is considered to have no effect on mechanical characteristics and strength properties of rock; filtration flow mainly forms on the low-permeable core of the dam. Usually, the dam core consists of soft (loess) soil, therefore its water saturation significantly influences physical and mechanical properties of soil. A two-dimensional problem is considered for the cross section of a dam using the equation of state with allowance for structural changes in rock fill and moisture content of a dam core with lowpermeable trapezium properties. For earth dams, as is known, a filtration flow is formed in the dam body. The problem is solved numerically - by the method of finite differences. The results are presented in the form of graphs and are analyzed.
\end{abstract}

\section{Statement of the problem}

The design and operation of earth dams located in seismic regions requires researchers to predict the dynamic behavior of these dams for various dynamic and seismic effects. Consequently, according to the dynamic behavior of dams, their bearing capacity, stability of slopes, etc., are determined. Dynamic methods for calculating earth dams, taking into account the wave nature of seismic effects, have been developed by many authors [1-2]. Considering soil as a two-phase medium, these studies take into account the water saturation of soil. However, the solutions of such problems for different dams are obtained at the initial moments of time. In [3-4], a dynamic method for calculating earth dams, with account of plastic and other soil properties, is developed and non-stationary problems are solved [5-6].

Let us consider a two-dimensional problem for a cross section of an earth structure (earth dam, pit walls, earth slopes, etc.) using nonlinear equations of state with allowance for structural changes and moisture content. Dynamic behavior and stress-strain state are studied on the example of the Charvak dam. Place the origin of the Cartesian coordinate system on the lower point of the upstream slope of the dam (pit walls, earth slopes, etc.) and direct the $O x$-axis along the base in the lower surface (Figure 1). Let a dynamic effect starts to act on earth structure from the base at the point in time $t>0$. If to take the acting action according to the record of a real velocigram (accelerograms or seismograms) of

\footnotetext{
*Corresponding author: khusanov@mail.ru
} 
earthquakes on the entire lower surface of the dam, then there is no need to study the behavior and operation of the base of the earth structure.

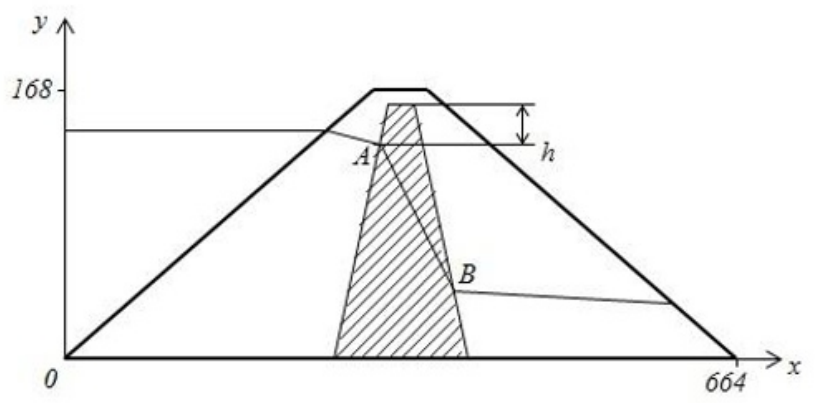

Fig. 1. Cross section of hydrotechnical structures.

Taking the acting effect on the entire lower surface of earth structure from the base, the system of equations in the Eulerian representation is integrated numerically by the finite difference method, including the equations of motion, continuity and the Cauchy relation for the soil structure:

equations of motion

$$
\rho \frac{d v_{x}}{d t}=\frac{\partial \sigma_{x x}}{\partial x}+\frac{\partial \tau_{x y}}{\partial y}+\rho \mathrm{F}_{x}, \quad \rho \frac{d v_{y}}{d t}=\frac{\partial \tau_{x y}}{\partial x}+\frac{\partial \sigma_{y y}}{\partial y}+\rho \mathrm{F}_{\mathrm{y}}
$$

continuity equation

$$
\frac{\partial v_{x}}{\partial x}+\frac{\partial v_{y}}{\partial y}=\frac{1}{V} \frac{d V}{d t}
$$

the relationship between the kinematic parameters, i.e. Cauchy relations in the form

$$
\dot{\varepsilon}_{x x}=\frac{\partial v_{x}}{\partial x}=\frac{\partial \dot{x}}{\partial x}, \quad \quad \dot{\varepsilon}_{y y}=\frac{\partial v_{y}}{\partial y}=\frac{\partial \dot{y}}{\partial y}, \quad \dot{\varepsilon}_{x y}=\frac{\partial \dot{y}}{\partial x}+\frac{\partial \dot{x}}{\partial y},
$$

where $\rho=\rho(x, y, t)$ is the density of the medium; $v_{x}=\dot{x}, v_{y}=\dot{y}$ are the components of velocity vector $\vec{v}=\vec{v}(x, y, t) ; \sigma_{x x}, \sigma_{x y}=\sigma_{y x}=\tau_{x y}, \sigma_{y y}$ are the components of the stress tensor $\hat{\sigma}=\hat{\sigma}(x, y, t) ; \quad V(x, y, t)$ is the relative volume, related to soil density; $\varepsilon_{x x}, \varepsilon_{x y}=\varepsilon_{y x}=\varepsilon_{x y}, \varepsilon_{y y}$ are the components of strain tensor $\hat{\varepsilon}=\hat{\varepsilon}(x, y, t) ; F_{x}, F_{y}$ are the projections of the density of external mass forces in the direction of the corresponding coordinates.

For the closure of the system of equations (1)-(3), the equation of state of earth structure should be added. The equations of state for shear strain of rock fill are taken in the form [79]: under loading

$$
\begin{gathered}
\frac{d P}{d t}=K\left(I_{S}\right) \frac{\dot{V}}{V} \\
\frac{d}{d t}\left(\frac{S_{x x}}{G\left(I_{S}\right)}\right)+\lambda \frac{S_{x x}}{G_{*}}=2\left(\dot{\varepsilon}_{x x}-\frac{1}{3} \frac{\dot{V}}{V}\right), \quad \frac{d}{d t}\left(\frac{S_{y y}}{G\left(I_{S}\right)}\right)+\lambda \frac{S_{y y}}{G_{*}}=2\left(\dot{\varepsilon}_{y y}-\frac{1}{3} \frac{\dot{V}}{V}\right),
\end{gathered}
$$




$$
\frac{d}{d t}\left(\frac{S_{x y}}{G\left(I_{S}\right)}\right)+\lambda \frac{S_{x y}}{G_{*}}=2 \dot{\varepsilon}_{x y}, \quad \frac{d}{d t}\left(\frac{S_{z z}}{G\left(I_{S}\right)}\right)+\lambda \frac{S_{z z}}{G_{*}}=2\left(0-\frac{1}{3} \frac{\dot{V}}{V}\right)
$$

under unloading

$$
\begin{gathered}
\frac{d P}{d t}=K \frac{\dot{V}}{V} ; \quad \frac{d S_{x x}}{d t}=2 G_{*} \exp \left(\alpha_{1}\right)\left(\dot{\varepsilon}_{x x}-\frac{1}{3} \frac{\dot{V}}{V}\right), \quad \frac{d S_{y y}}{d t}=2 G_{*} \exp \left(\alpha_{1}\right)\left(\dot{\varepsilon}_{y y}-\frac{1}{3} \frac{\dot{V}}{V}\right), \\
\frac{d S_{x y}}{d t}=2 G_{*} \exp \left(\alpha_{1}\right) \dot{\varepsilon}_{x y}, \quad \frac{d S_{z z}}{d t}=2 G_{*} \exp \left(\alpha_{1}\right)\left(0-\frac{1}{3} \frac{\dot{V}}{V}\right)
\end{gathered}
$$

Equation of state for the dam core with account of moisture content is [9-11]:

$$
\begin{gathered}
\frac{d P}{d t}=K_{s a t} \exp \left(\alpha_{K}\left(1-I_{W}\right)\right) \frac{\dot{V}}{V} ; \quad \frac{d S_{x x}}{d t}+\lambda_{0} S_{x x}=2 G_{s a t} \exp \left(\alpha_{G}\left(1-I_{W}\right)\right)\left(\dot{\varepsilon}_{x x}-\frac{1}{3} \frac{\dot{V}}{V}\right) \\
\frac{d S_{y y}}{d t}+\lambda_{0} S_{y y}=2 G_{s a t} \exp \left(\alpha_{G}\left(1-I_{W}\right)\right)\left(\dot{\varepsilon}_{y y}-\frac{1}{3} \frac{\dot{V}}{V}\right) \\
\frac{d S_{z z}}{d t}+\lambda_{0} S_{z z}=-\frac{2}{3} G_{s a t} \exp \left(\alpha_{G}\left(1-I_{W}\right)\right) \frac{\dot{V}}{V}, \quad \frac{d S_{x y}}{d t}+\lambda_{0} S_{x y}=2 G_{s a t} \exp \left(\alpha_{G}\left(1-I_{W}\right)\right) \dot{\varepsilon}_{x y}
\end{gathered}
$$

where under plastic strain $\lambda_{0}=\frac{3 \Omega}{2 Y^{2}(P)}$ is non-zero. Here, the stress tensor components are expressed in terms of the components of the deviator and the spherical part of the tensor in the form

$$
\sigma_{x x}=S_{x x}-P, \quad \sigma_{y y}=S_{y y}-P, \quad \sigma_{z z}=S_{z z}-P .
$$

Closed system of equations (1)-(7) has 11 equations relative to 11 unknowns $\left(v_{x}, v_{y}\right.$, $\left.V=\rho_{0} / \rho, \dot{\varepsilon}_{x x}, \dot{\varepsilon}_{x y}, \dot{\varepsilon}_{y y}, P, S_{x x}, S_{x y}, S_{y y}, S_{z z}\right)$. This system of equations is solved with regard to the initial conditions: at $t=0$ in the whole area of earth structure

$$
\begin{gathered}
v_{x}=0, \quad v_{y}=0 ; \quad V=1, \quad \dot{\varepsilon}_{x x}=0, \quad \dot{\varepsilon}_{x y}=0, \quad \dot{\varepsilon}_{y y}=0 ; \\
P=0, \quad S_{x x}=0, \quad S_{x y}=0, \quad S_{y y}=0, \quad S_{z z}=0
\end{gathered}
$$

and boundary conditions at $t>0$. The boundary conditions are formulated in the following way: on the bottom surface $(y=0)$ of the dam, a seismic load is set, varying over time in the form of a harmonic function of time; on the slopes and crest of the dam the stress free conditions are accepted. At the boundary between the dam core and the rock fill the conditions of complete adhesion are accepted.

Thus, the system of equations (1)-(7) with initial and boundary conditions determines the dynamic behavior and stress-strain state of earth structures under static and dynamic loads. Note that the external dynamic load is expressed as boundary conditions. In the case of account of own weight (in the presence of mass forces), these loads enter directly the equations (1). 


\section{Method}

The problem is solved by the finite difference method using the Wilkins scheme for twodimensional problems. The finite-difference relations of the considered equations are available in $[3,12]$. Using the methodology $[3,12-13]$, the problem is solved numerically. The physicomechanical parameters of the problem are assumed as follows: the initial density of the dam rock fill is $1980 \mathrm{~kg} / \mathrm{m} 3$, the initial density of core material at natural moisture content is $1700 \mathrm{~kg} / \mathrm{m} 3$; strength indices of stone fills are equal $\mathrm{E}=6,21 \mathrm{GPa}$, $v=0,25, \mathrm{Y}_{0}=0,6 \mathrm{MPa}, \mu=0,4, \mathrm{Y}_{\mathrm{PL}}=12 \mathrm{MPa}, \alpha=1,5$, respectively; the corresponding parameters for the dam core are: $\mathrm{K}_{\mathrm{sat}}=5 \mathrm{MPa}, \mathrm{G}_{\mathrm{sat}}=0,96 \mathrm{MPa}, \mathrm{c}_{\mathrm{sat}}=0,05 \mathrm{MPa}$, $\mu_{\text {sat }}=0,24, \alpha_{\mathrm{k}}=2,5, \alpha_{\mathrm{G}}=4,02, \beta=3,92, \gamma=1,09, \mathrm{~W}_{0}=13 \%$ and $\mathrm{W}_{\text {sat }}=38 \%$. The coordinates of points of filtration flow $\mathrm{A}$ and $\mathrm{B}$ in Figure 1 are considered to be equal: $\{336$ $\mathrm{m} ; 157,71 \mathrm{~m}\}$ and $\{387,5 \mathrm{~m} ; 41,14 \mathrm{~m}\}$. Numeric results are given in the form of graphs.

\section{Numerical results and analysis}

Consider the stress-strain state of an earth dam under the action of load in vertical direction only in the absence of mass forces. Let's assume, that under the action of a seismic load, the bases of the dam move in a sinusoidal manner with amplitude of $0.15 \mathrm{~m}$ and an oscillation period of 2 seconds.

Plastic flow zones, i.e. the zones where $I_{s}=1$, under the action of harmonic load are shown in Figure 2. It is seen that plastic strains are experienced by the particles located in the upstream and near-crest parts of the dam. Figure 3 shows the changes in shear strains over time at the points 1, 2, 3 and 4 (respectively, with coordinates $\{329,127\}$ located in the near-crest upstream slope zone; $\{98,37\}$ - upstream slope zone at the base; $\{342,37\}$ in the core center at the bottom of the base and $\{342,80\}$ in the core center of the dam). As seen from Figure 3, significant shear strains occur in the central part of the dam, i.e. in the core of the dam, where the strength characteristics are much smaller than in the rock fill slope zones.

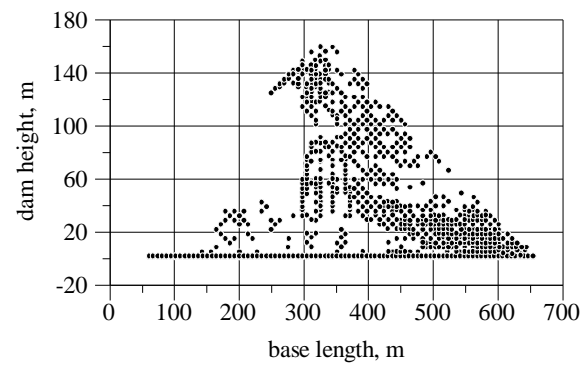

Fig. 2. Plastic strain zone in the dam body.

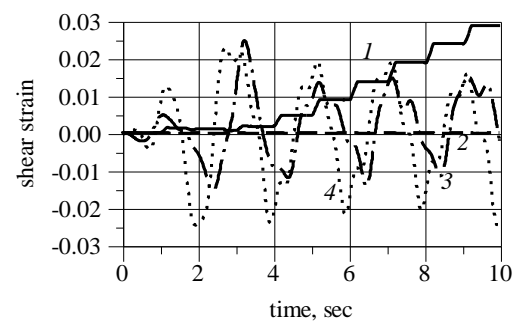

Fig. 3. The change in shear strain over time.

Figure 4 shows the changes in stresses over time at points 1, 2, 3 and 4. Significant stresses are observed in the rock-filled parts of the dam compared to the "soft" core of the dam. At points 1 and 2, located in the slope zones, multiple reflections of waves are observed from the free slope surfaces. In the case of vertical longitudinal stresses (Figure 4), all stresses are comparable, i.e. the difference in values is small.

The stress state of earth dam to set the vertical displacement of the dam base with doubled frequency is presented in Figure 5. The case of two times increase in the frequency of the specified displacements correspond to the stress-strain states presented in Figures 68. 

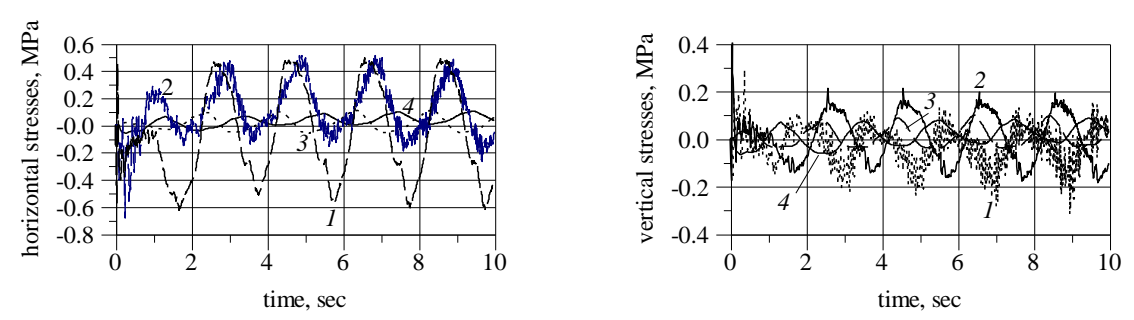

Fig. 4. Stress variation over time.
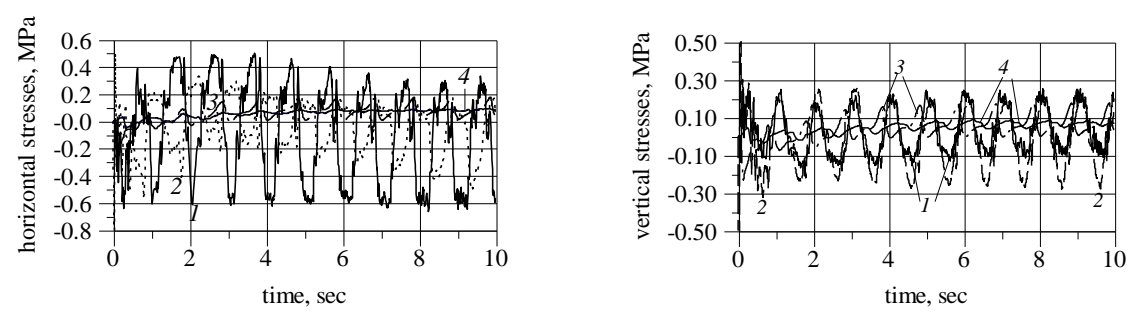

Fig. 5. Stress variation over time.
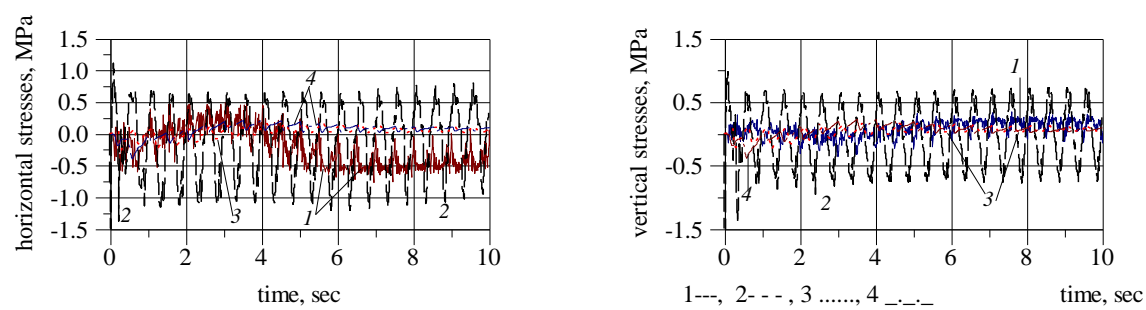

Fig. 6. Stress variation over time.
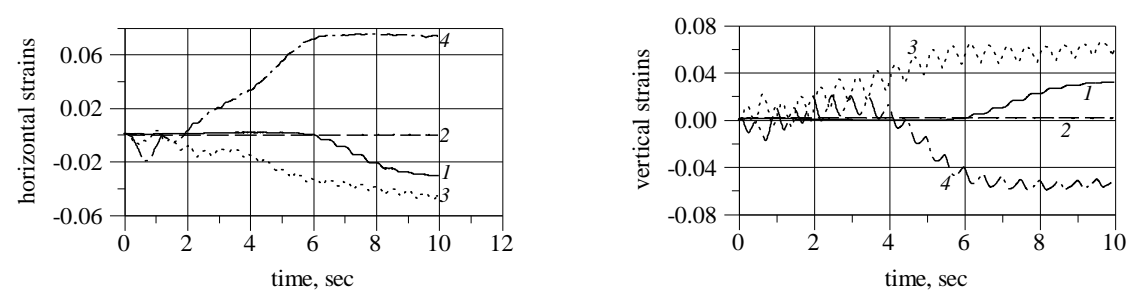

Fig. 7. Change in strain over time.

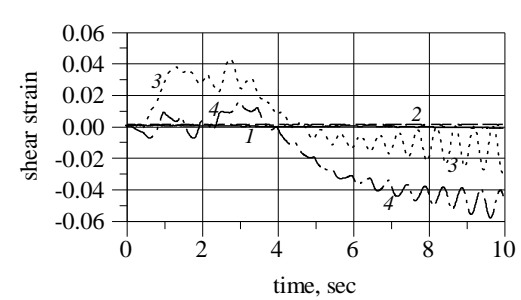

Fig. 8. The change in shear strain over time.

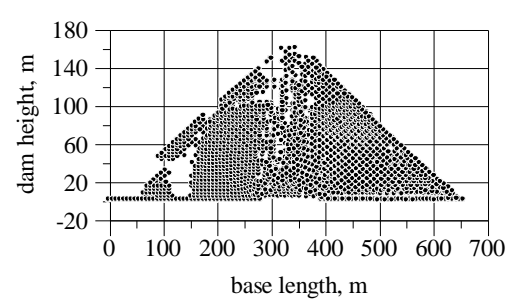

Fig. 9. Plastic strain zone in the dam body.

It is also seen that the changes in the longitudinal and shear strains over time of the considered points 1, 2, 3 and 4 (respectively, curves 1-4) occur in the central part of the dam, i.e. in the core of the dam, where the strength characteristics are much smaller than in the rock fill slope zones. Zones of plastic flows under harmonic load are shown in Figure 9. 
It shows that plastic strains are experienced by almost all dam particles in cases of an increase in the frequency of external load.

Consider the task for the same earth dam, when seismic load is set on the bottom surface $(y=0)$ of the dam, varying over time in the form of a real velocigram recorded during the earthquakes (Figure 10); the slopes and crest of the dam are stress-free [14]. The results of these calculations are presented in the form of graphs in Figure 11.

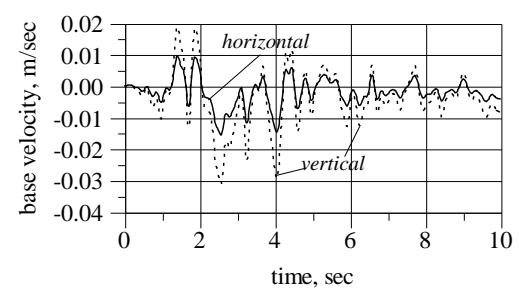

Fig. 10. Earthquake velocigram.

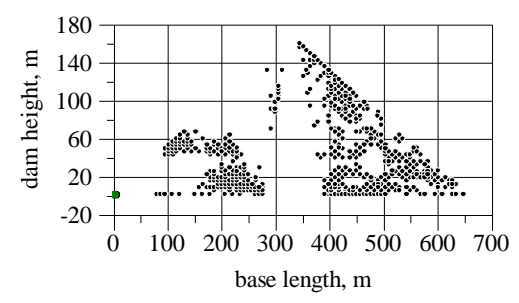

Fig. 11. Plastic strain zone in the dam body.

As expected, maximum stresses under seismic effect occur on rock fills of the dam. At the same time, the values of horizontal stresses are approximately two times greater than vertical ones. By reducing the strength characteristics of the dam core, the stress state varies within $0.05 \mathrm{MPa}$.

\section{Discussion}

The stresses arising in calculations under the effect of velocigram record (Figure 10) are permissible for the operation of the dam in question. However, in the crest and slope zones, the stress values from time to time change to a positive value. Consequently, a tension occurs in these places. As is known, soil resists tension only at low stress values. It is possible that the resulting stresses can overpass the allowable stress of soil under tension. Plastic strain begins at the crest zone (due to the free crest). At $t=2-3 \mathrm{sec}$, the area of plastic strain practically covers the largest upstream slope area. Over time, the occurrence of out-of-limit states in dam particles decreases (Figure 11).

\section{Conclusions}

The stress-strain state at the initial time, approximately at $\mathrm{t}=2 \mathrm{sec}$, is the most unfavorable state. High values of stresses and residual strains, as well as displacements in the slope and near-crest zones of the dam can lead to the destruction of the crest or slope zones of the dam. High-frequency loads accompany the increase in stresses and the emergence of vast zones of out-of-limit conditions of the earth dam.

The research is conducted under financial support of the Ministry of Innovation Development of the Republic of Uzbekistan on the project FA-Atex-2018-(1+2).

\section{References}

1. A. Goldin, L. Rasskazov, Design of soil dams (Moscow, DIA, 2001)

2. Yu.K. Zaretsky, V.N. Lombardo, Statics and dynamics of earth dams (Moscow, Energoatomizdat, 1983)

3. B.E. Khusanov, K.D. Salyamova, Dokl. Acad. Sci. Rep. of Uzb. 9, 12 (1998) 
4. K.D. Salyamova, B.E. Khusanov, Trans. of Acad. of Sci.of Azerbaijan. Ser. Phys. \& Math. Sci. 3, 260 (1998)

5. K. Terzaghi, Theoretical Soil Mechanics (John Wiley \& Sons, 2001)

6. F. García, J.J. Aznárez, L.A. Padrón, O. Maeso, Soil Dyn. \& Eart. Eng. 90, 442 (2016)

7. K.S. Sultanov, B.E. Khusanov, Uzb. J. Prob. Mech. 4, 16 (2000)

8. B.E. Khusanov, XI All-Russian Congress on Fundamental Problems of Theoretical and Applied Mechanics, Collection of reports (Kazan University Press, 2015)

9. K.S. Sultanov, B.E. Khusanov, Soil Mech. \& Found. Eng. 3, 80 (2001)

10. B.E. Khusanov, Trans. of Acad. of Sci.of Azerbaijan. Ser. Phys. \& Math. Sci. 1, 199 (2001)

11. K.S. Sultanov, B.E. Khusanov, Uzb. J. Prob. Mech. 5, 27 (2004)

12. M.L. Wilkins, Computer Simulation of Dynamic Phenomena (Springer-Verlag, 2010)

13. K.S. Sultanov, B.E. Khusanov, Soil Mech. \& Found. Eng. 3, 81 (2002)

14. B.S. Nurtaev, Initiation of seismic effect to calculate the reliability of high earth dams (Moscow, Institute of Earth Physics, 1990) 